\title{
Domenico Cirillo
}

Ein Lebenslauf, 1739-1799

Von Johann Ulrich Marbach

Domenico Cirillo wurde am 10. April 1739 im Dorfe Grumo, nördlich von Neapel, als Kind wohlangesehener Eltern geboren. Die Familie hatte bereits ungewöhnliche Geister hervorgebracht. Der Vater Innocenzio war ein Neffe von Nicola Cirillo ${ }^{1}$, die Mutter, eine Capasso, Nichte von Nicola Capasso ${ }^{2}$. Frühreif und mit glänzenden Geistesgaben ausgestattet, erweckte Domenico Hoffnungen. Er war noch ein Kind von sieben Jahren, als er nach Neapel zu seinem Onkel Sante Cirillo (Salvator Cyrillus) kam, der damals dort ein bekannter Naturforscher war. Der Onkel nahm sich der Erziehung des Knaben liebevoll an. In einem Rechenschaftsbericht an die Eltern konnte er von erstaunlichen Fortschritten des Zöglings berichten.

Gute Lehrer unterrichteten Cirillo in Griechisch und Latein. Schritt für Schritt wurde er in die Philosophie und in die mathematischen Fächer eingeführt. Auch lernte er zeichnen. Die Stunden des Tages wurden sorgfältig eingeteilt und nützlich verwendet.

Von kindlicher Neugierde getrieben, leistete der Knabe zuweilen seinem Onkel Gesellschaft, wenn dieser sich dem botanischen Garten widmete, den ihm sein Onkel Nicola Cirillo hinterlassen hatte. Dabei keimte in dem ;naben eine Neigung für die Naturwissenschaften. Er hielt sich nun ständig in der Nähe des Onkels, wenn dieser mit den Pflanzen beschäftigt war, die er hegte oder auch pflückte, um sie zu zeichnen, zu klassifizieren und dem Herbarium einzuverleiben. Indem der Knabe an solchen Arbeiten teilnahm, gelangte er allmählich von der bloßen Neugierde zur genauen Kenntnis der Dinge.

Sante Cirillos Schüler, Mitarbeiter und Freunde in der Botanik waren Gelehrte wie Nicola Pacifico, Angiolo Fasano, Natale Lettieri ${ }^{3}$, Nicola Braucci ${ }^{4}$ und Vincenzo Petagna ${ }^{5}$. Sie verwunderten sich über den aufgeweckten Geist, den Scharfsinn und den Ernst des kleinen Domenico, der mit seinen Äußerungen seinem Alter weit vorauseilte. Darüber freute sich der Onkel, und da er bemerkte, daß der Verstand seines Neffen schon hinreichend gefestigt war, begann er ihm in Gesprächen die ersten theoretischen Kenntnisse der Wissenschaft mitzuteilen und schließlich das ganze Tournefortsche System zu erklären ${ }^{6}$. 
Francesco Serao ${ }^{7}$ war ein Schüler Nicola Cirillos gewesen und bewahrte für diesen Dankbarkeit und Verehrung. Er war gewohnt, im Hause ein und aus zu gehen und lange Gespräche mit Sante Cirillo zu führen. Es ergab sich von selbst, daß er mit Anteilnahme die Entwicklung des jungen Cirillo verfolgte. Und es fiel ihm nicht schwer, in dem Heranwachsenden auch eine Begeisterung für die Medizin zu wecken. Daher begann Domenico unter Zustimmung seines Onkels mit dem Studium dieser Wissenschaft.

Verblüffend schnell durchlief er den ganzen Universitätskurs an der Königlichen Hochschule in Neapel. Daneben jedoch setzte er seine botanischen Studien fort. In seinem einundzwanzigsten Lebensjahr meldete er sich 1760 für den Lehrstuhl der Botanik und wurde nach dem öffentlichen Wettbewerb einstimmig gewählt. Damals nahm diese Wissenschaft gerade einen Aufschwung. Als erster lehrte Cirillo in Neapel Linnés berühmtes System.

1764 unternahm er eine Reise nach Sizilien, da jene Insel einen Überfluß an seltenen Gewächsen bietet. Mit unermüdlichem Eifer sammelte er alles, was er an ihm noch unbekannten Pflanzen fand. Mit diesen bevölkerte er den botanischen Garten, den Nicola Cirillo gegründet hatte. Bis 1766 widmete er sich wieder seiner Lehrtätigkeit. Aber sein Forscherdrang ließ ihm keine Ruhe. Es trieb ihn immer wieder hinaus ins Freie, wo er die Pflanzen an ihren ursprünglichen Standorten aufsuchte. So entschloß er sich zu einer zweiten Reise. Zusammen mit dem englischen Naturwissenschaftler Simons durchstreifte er Apulien, Kalabrien und die Gegend von Otranto. Ein Jahr darauf nahm er seinen besten Freund Nicola Pacifico mit auf eine Exkursion in die Abruzzen. Seine Wanderungen kreuz und quer durch das Königreich Neapel hat er beschrieben. Als er sich bereits im Ausland einen Namen gemacht hatte, reiften in ihm Pläne, auch größere Reisen zu unternehmen. Milady Walpole bot ihm 1769 eine Möglichkeit an, in ihrer Gesellschaft nach Paris zu fahren. Da sein Ruf ihm vorausgeeilt war, konnte er dort Schriftsteller und Gelehrte (Buffon, d'Alembert, Diderot, Abbé Nollet) kennenlernen. Benjamin Franklin, der sich damals in Paris aufhielt, schloß ihn ins Herz und wechselte später mit ihm Briefe. Bei soviel Aufmunterung fiel ihm der Entschluß, auch England zu besuchen, nicht schwer. Dort empfing ihn John Pringle $^{8}$, der den Sohn Neapels aus seiner Tätigkeit bereits kannte. Cirillo benutzte in London die Gelegenheit, an einem Sezierkurs teilzunehmen und seine anatomischen Kenntnisse zu erweitern. Seine besondere Aufmerksamkeit erregte die Einspritzungsmethode in die Lymphgefäße, die William Hunter an der Universität ausübte. Unter Berücksichtigung jener Erkennt- 
nisse schrieb Cirillo später ein Buch über die Geschlechtskrankheiten, besonders die Syphilis, das ins Deutsche und Französische und auch ins Russische übersetzt wurde. Jene Arbeit zum Wohle der Kranken verhalf der medizinischen Wissenschaft Neapels zu neuem Ansehen.

In London fehlte Cirillo kaum je bei den Konversationsabenden, die Pringle in seinem Hause veranstaltete und zu denen sich mehrere Gelehrte einfanden. Bei diesen Anlässen traten Cirillos Kenntnisse so vorteilhaft hervor, daß alle ihn drängten, eine Denkschrift in englischer Sprache abzufassen und der Königlichen Gesellschaft der Wissenschaften in London einzureichen. Er tat es, und die Royal Society ernannte ihn zum korrespondierenden Mitglied. Nach einem Aufenthalte von anderthalb Jahren zog er wieder nach Frankreich, wo er Benjamin Franklin wiedersah. Mit Pringle, Hunter und anderen blieb er in freundschaftlichem Briefwechsel verbunden.

1770 kehrte Cirillo nach Neapel zurück. Dabei machte er in den wichtigsten Städten Italiens halt und lernte weitere Gelehrte kennen. Auch mit ihnen knüpfte sich ein Briefverkehr an. Durch viele neue Erfahrungen bereichert, wußte er seiner Heimat noch besser zu dienen und erwarb das allgemeine Vertrauen. In der Ausübung des ärztlichen Berufes hatte er Neues zu bieten. Vor allem verwertete er seine Kenntnisse in der Chemie und Botanik zur Zubereitung von Arzneien für die Behandlung verschiedener Krankheiten. Gleichzeitig gab er sein Wissen mit fabelhafter Lehrbegabung weiter, so daß ihm Lernende zuströmten.

Als am Spital Santa Casa degli Incurabili die Lehrstelle frei wurde, die der Physiologe Orazio Biancardi innegehabt hatte, wurde Cirillo damit betraut. Hier bewährte sich abermals seine Begabung, indem er die Physiologie in neuem Lichte darstellte. Dies zog eine Schar von Studenten nach Neapel. Cirillo war auch der erste, der hier öffentlichen Unterricht in der Geburtshilfe erteilte und diesem Lehrgebiet Ansehen gab. Eine falsche Scham bei den Frauen und ein eingewurzeltes Vorurteil hatten diesen Beruf bisher fast ganz in den Händen von Hebammen belassen, die oft beschränkt und abergläubisch waren. Unter Cirillos Schülern in der Geburtshilfe zeichneten sich später vor allem Bruno Amantea ${ }^{9}$ und Angelo Leonessa aus. In Anerkennung seiner Bemühungen ernannten die Spitalvorsteher Cirillo mit ehrenvollem Stimmenmehr zum Chefarzt des Krankenhauses.

Damals war in Neapel der chinesische Arzt Hivi-Kiou angekommen. Er übte eine neue Heilpraxis aus, die Sfigmika oder Lehre vom Puls. Nicht leicht gewährte er Zutritt, und nur auf ausdrückliche Erlaubnis des Oberhauptes der Chinesen in Neapel erteilte er mit Hilfe eines Dolmetschers dem 
einen oder anderen Kranken Rat. Eine Dame wünschte von Cirillo, daß er sie zu ihm begleite. Der chinesische Doktor untersuchte ihren Puls und diagnostizierte ihr Leiden. Nun ließ sich auch Cirillo den Puls fühlen. Der Arzt erklärte, er müsse in frühen Jahren einmal Herzbeschwerden gehabt haben. Cirillo konnte sich an nichts derartiges erinnern; seine Mutter erklärte jedoch, daß diese Feststellung zutreffe. Da Hivi-Kiou nicht ein Wunderdoktor war, sondern wirkliche Kenntnisse besaß, kehrte Cirillo öfters zu ihm zurück, um sich mit ihm zu unterhalten, solange der chinesische Arzt sich in Neapel aufhielt. Cirillo ging der Pulslehre auf den Grund und begann sie anzuwenden. Die Pulslehre des Hippokrates und Galen, die aus uralten Quellen geschöpft war, erschien ihm in neuer Bedeutung.

Beeindruckt von den diagnostischen Verfahren des Altertums, wandte sich Cirillo von der spekulativen Medizin ab und förderte die exakte klinische Beobachtung. Seine Krankenjournale wurden berühmt. Wenn er sich den Kranken näherte, um ihre Krankengeschichte aufzuzeichnen, umringten ihn lerneifrige Schüler und Ärzte. Cirillo notierte genau die Symptome und den bisherigen Verlauf der Krankheit, ferner die anzuwendenden Mittel und die Art der Behandlung. In den folgenden Tagen zeichnete er auf, wie die Arzneien je nach ihrer Menge wirkten und was sonst noch zu bemerken war. Nach der Heilung beschrieb er die verschiedenen Zustände bis zur Wiederherstellung. Wenn dagegen ein Kranker starb, so öffnete er den Leichnam und merkte sich das Auffallende. Mißgriffe in Diagnosen und Verordnungen hielt er mit unnachahmlicher Offenheit und strenger Sachlichkeit in seinem Tagebuch fest. Die Todesursache brachte er mit den zuvor angestellten Beobachtungen in Zusammenhang. Daß dieses klinische Manuskript nie gedruckt erschien, wurde von allen gelehrten Ärzten beklagt.

Als Cirillos Ruf sich in Europa verbreitete, zog gleichzeitig noch ein anderer Stern über Neapels Horizont auf: Domenico Cotugno ${ }^{10}$. Dieser Arzt stach unter seinen Kollegen ähnlich wie Cirillo hervor. Trotzdem wurden die beiden nicht Nebenbuhler. Freunde waren sie schon in ihren Jünglingsjahren gewesen. Verwandte Neigungen führten sie näher zusammen. In ihrer gegenseitigen Achtung und Zuneigung gaben sie ein nachahmenswertes Beispiel menschlicher Würde. Durch den Grafen von Firmian erging an beide gleichzeitig eine Berufung an die Universität Pavia. Da sie mit vorteilhaften Bedingungen verknüpft war, hätte die Versuchung nahegelegen, ein so großzügiges Angebot anzunehmen. Beide wiesen es entschlossen zurück, um auch weiterhin ihrer Vaterstadt zu dienen.

Als aber durch den Tod Angelo de Robertis der Lehrstuhl für angewandte 
Medizin an der Königlichen Hochschule in Neapel frei wurde, bewarb sich Cirillo darum. Der rührige Justizminister Marchese de Marco, dem gleichzeitig die Verantwortung für die kulturellen Belange des Königreichs übertragen war, sah voraus, daß Cirillo den Wettbewerb gewinnen werde. Um zu verhindern, daß durch das Umsatteln dem botanischen Lehrstuhl die Leuchte entzogen werde, bat er Cirillo zu sich und ließ seine Überredungskunst spielen, um ihn von der Teilnahme am Concorso abzuhalten. Er versprach ihm eine Erhebung des botanischen Lehrstuhls zu einer «Cattedra primaria col soldo», und die Entschädigung sollte von 150 auf 500 Dukaten steigen. Doch die innere Entwicklung hatte Cirillo immer mehr zur Medizin geführt. So aufrichtig er dem Minister seine Erkenntlichkeit zu verstehen gab, lehnte er dennoch das Angebot mit bescheidenen Worten ab. Nachdem der Marchese auch mit Bitten nicht zum Ziele gelangt war, steckte er sich hinter Francesco Conforti ${ }^{11}$ und hoffte, dieser könne Cirillo überreden. Als Cirillo sich so unter Druck gesetzt sah, erklärte er dem Freunde, wenn er sich vom Wettbewerb zurückziehen müsse, bitte er den Minister um die Erlaubnis, auch vom Lehrstuhl der Botanik abtreten zu dürfen. Diese unmißverständliche Antwort genügte, und Cirillo wurde beim Wettbewerb auf den Lehrstuhl der Medizin gewählt.

Seine Lehrtätigkeit stärkte das Ansehen der Universität. Die Studenten drängten sich in seinen Hörsaal. Auch aus entlegenen Provinzen und sogar aus dem dicht mit Hochschulen besetzten Oberitalien strömten Schüler herbei. Nicht einmal in Pavia, wo ausgezeichnete Lehrer wirkten, könne man so zuträgliche Vorlesungen hören, behaupteten Studenten, die von dort kamen. Sein herzliches Wesen und seine anmutig-witzige Weise erweckten Vertrauen, Ehrerbietung und freundschaftliche Gefühle. Als Arzt hatte er Einfühlungsgabe. Seine botanischen und chemischen Kenntnisse und seine Spitalerfahrung baute er geschickt in seine Darlegungen ein. Bei den Fremden, die Neapel besuchten, wurde es Mode, einer Vorlesung Cirillos beizuwohnen. Man hörte, wie eines Tages Graf Brunswick zu ihm sagte: «Ich habe die göttliche Medizin immer hochgeschätzt; nie aber verspürte ich Lust, Arzt zu werden, - bis ich Sie und Cotugno gehört habe.»

Die Berühmtheit des Lehrers kam auch seiner Arztpraxis zugute. Die Stunden des Tages reichten nicht aus, der Nachfrage gerecht zu werden. Zudem ging er lieber zu den Armen als zu den Reichen. Es kamen Kranke aus ganz Italien und aus dem Ausland. Sogar aus Boston reiste eine wichtige Persönlichkeit eigens her, um von einem chronischen Leiden erlöst zu werden. Auch Sir William Hamilton, der englische Botschafter in Neapel, 
ließ sich von Cirillo behandeln und empfahl ihn seinen Landsleuten. Cirillos Haus überbordete von Unglücklichen, die Heilung suchten. Noch lange nach seinem Tode erinnerte man sich in Neapel an heikle Krankheitsfälle, die durch die Kunst des großen Arztes eine glückliche Wendung genommen hatten.

Als Königin Karolina von Neapel die Malerin Angelika Kauffmann ${ }^{12}$ nach Neapel rief, damit sie die königliche Familie porträtiere, lernte diese Cirillo kennen. Angelika Kauffmann war die Tochter eines Vorarlberger Malers und einer Schweizerin aus Chur. Was sie nach dem Urteil bedeutender Zeitgenossen auszeichnete, war ein kindlich reines Gemüt und die Fähigkeit, eine seelenvolle Atmosphäre um sich zu verbreiten (Herder). Bevor sie Neapel verließ, malte sie Cirillos Bildnis. Es gelangte später in den Besitz des Appellationsrichters Giuseppe Castaldi.

Die Liebe zu den Pflanzen verließ Cirillo nie. Er fühlte sich mit jenen seiner Schüler am meisten verbunden, die sich ebenfalls dieser Wissenschaft hingaben. Auf eigene Kosten sandte er sie in verschiedene Teile des Reiches, um sie in ihrem Studium zu ermuntern und den Bestand seines botanischen Gartens zu bereichern. So schickte er Saverio Macrì in die Gegend von Majella, später nach Capri und zusammen mit Francesco Filomena ${ }^{13}$ auf das Vorgebirge von Sorrent. Matteo Tondi ${ }^{14}$ und Giovanni Sasso stiegen zum Monte Vergine hinauf. Gaetano Nicodemi ${ }^{15}$ reiste nach Apulien bis zum Gargano und nach Sizilien. Im Gebiet um Capua und in den Pontinischen Sümpfen botanisierte Nicodemi zusammen mit Domenico Siciliani. Im Cilento und längs der amalfitanischen Küste begleiteten ihn Macrì und Cirillos Lieblingsschüler, der begabte Francesco Ricca. Jedes Jahr schickte Cirillo Geld an Manni in Taranto, der für ihn Insekten sammelte. Von diesen Exkursionen floß ihm eine reiche naturgeschichtliche Ausbeute zu. Sie erweiterte seinen botanischen Garten, worin er mit unausgesetztem Fleiß und unter beträchtlichen Kosten nahezu sechstausend Pflanzen hegte und pflegte. Gleichzeitig stand er mit hervorragenden Botanikern in Verbindung, so mit Linné, der nach ihm die Pflanzenfamilie der Cirillaceen taufte. Peder Ascanius ${ }^{16}$ und Johann Anders Murray ${ }^{17}$, zwei der angesehensten Schüler Linnés, reisten nach Neapel, um Cirillos Bekanntschaft zu machen. Als die beiden Männer das Museum des Gelehrten betraten und ihnen das herrliche Herbarium von Ferrante Imperato ${ }^{18}$ gezeigt wurde, kniete Murray in einer Aufwallung von Begeisterung davor nieder und küßte es.

So war Cirillos Leben erfüllt von seinen Taten als Forscher, Sammler, Lehrer, Arzt und Helfer der Armen. Stark war sein Verantwortungsbewußt- 
sein gegenüber dem öffentlichen Wohle. Dem Zeitgeist gegenüber war er in humaner und liberaler Art aufgeschlossen. Er verehrte Rousseau und hat Stücke aus dessen «Spaziergängen» ins Italienische übersetzt. Von den Errungenschaften der Neuzeit erhoffte er einen Fortschritt für sein eigenes Vaterland. Doch gerade diese Erwartung wurde ihm zum Verhängnis.

Neapel nahm am Krieg gegen das revolutionäre Frankreich teil. Ein französisches Heer stürzte das bourbonische Königtum und errichtete im Januar 1799 die Parthenopäische Republik. Cirillo, durch seinen Kampf gegen die Mißstände im Spital- und Gefängniswesen bekannt, wurde wider seinen Willen, da er sich ganz den Kranken widmen wollte, in den gesetzgebenden Rat gewählt. Gegen das Elend gründete er eine Hilfskasse, der er seine eigenen Ersparnisse zuwies. In jeder Straße sorgte er für einen Vater und eine Mutter der Armen. Doch schon im Sommer wurde durch die Armee des Kardinals Ruffo mit Unterstützung der englischen Flotte die Monarchie wiederhergestellt. Der 60jährige Cirillo hatte am Widerstand teilgenommen. Nelson, der nachmalige Held von Trafalgar, ließ vorsorglich gleich seinen gefährlichsten Gegner zur See, den Admiral Francesco Caracciolo, am Hauptmast seines Admiralsschiffes aufknüpfen ${ }^{19}$. Es geschah am 29. Juli 1799. Dann folgte eine Hinrichtung nach der andern bis in das Jahr 1800 hinein. Der erste Geschichtsschreiber der Revolution von 1799, Vincenzo Cuoco, zählt 120 Hinrichtungen auf: Gelehrte, Professoren, Ärzte, Priester, Bischöfe, hohe Offiziere, Juristen, Schriftsteller, Staatsmänner, Politiker, Kaufleute, Verleger, Frauen, Bürger, Adelige, Ingenieure, Bauern. Die rachsüchtige Königin Karolina trieb zu den Todesstrafen an und genoß die Beihilfe ihres Gemahls, des nichtsnutzigen Königs Ferdinand I., und das Einverständnis des englischen Machthabers Nelson. Cirillo wurde von einem Sondergericht verurteilt und zusammen mit den Patrioten Mario Pagano, Vincenzo Russo und Ignazio Ciaja am 29. Oktober 1799 auf dem Marktplatz Carmine, der Richtstätte Neapels, gehängt.

Nicht der kühne Heerführer Kardinal Ruffo war der Hauptschuldige an dieser Tragödie. Er hatte den Patrioten, als sie zur Übergabe der Stadt gezwungen waren, sichere Haft und Befreiung versprochen und kämpfte erbittert um das gegebene Wort, wurde aber übergangen. Auch die vielen neapolitanischen Anhänger der Monarchie waren nicht schuld, sondern das Königspaar und die Vertreter der englischen Macht ${ }^{20}$.

Noch am Hinrichtungstage wurde der Palazzo Cirillos mit den Sammlungen, Herbarien, Büchern, Manuskripten und Briefen zur Plünderung freigegeben. Unschätzbare Werte gingen verloren, darunter die Briefe Isaac 
Newtons an Nicola Cirillo. Unterdessen fuhr der König fort, in sein Tagebuch Aufzeichnungen über seine Verdauung und seine Kaninchenjagd einzutragen. Die Königin schlürfte beruhigt aus niedlichen Capodimontetäßchen die braune Schokolade. Zur Zerstreuung entnahm sie ihrer Bücherei eine Lektüre, die dem Geschmack eines lüsternen Dienstmädchens Ehre gemacht hätte. (Vergleiche das Bibliotheksverzeichnis, das Benedetto Croce veröffentlicht hat!)

Bei der Kunde von den Hinrichtungen empörte sich Europa, und im englischen Parlament kam es zu heftigen Auseinandersetzungen. Neapel hatte einen seiner größten Naturforscher und Ärzte für immer verloren. Als Held starb nicht so sehr Nelson als sein wehrlos gemachtes und schimpflich umgebrachtes Opfer: Domenico Cirillo ${ }^{21}$.

\section{Anmerkungen}

1 Nicola Cirillo (1671-1734), aus Grumo bei Neapel, Leibarzt des Papstes Innozenz XII., Professor der Physik und Medizin in Rom, dann in Neapel. Verfasser physikalischer und medizinisch-therapeutischer Abhandlungen in lateinischer Sprache: über das Quecksilber, über das Eisen, über das Erdbeben von 1731 in Apulien und im Königreich Neapel (Philosophical Transactions 1733); im gleichen Band erschienen seine Witterungsbeobachtungen seit 1718. Als Vorläufer empfahl er die Kaltwasserbäder bei Fieber. Das Hauptwerk erschien nach seinem Tode: «Consulti medici», 3 Bände, Neapel 1738; Venedig 1741. Zuvorderst steht seine Biographie, verfaßt von Francesco Serao: Nicolai Cyrilli, in Regia Universitate Neapolitana primarii medicinae practicae professoris et Regiae Societatis Londinensis socii, vita (mit Porträt).

2 Nicola Capasso (1671-1745), aus Grumo, Jurist, Gelehrter und Dichter in neapolitanischer Mundart. Seine Lehrer waren der Zivilrechtler und Altphilologe Domenico Aulisio und der Kirchenrechtler Girolamo Cappello. Er wurde 1703 als Professor Nachfolger Cappellos, 1717 Nachfolger Aulisios. Sein Anliegen war die Stärkung der weltlichen Regierung zur Sicherung des Friedens. Er übersetzte einen großen Teil von Homers Ilias in die neapolitanische Mundart. Ein Spätwerk war seine Tragödie «L'Otone» über Kaiser Otto III. Giambattista Vico und Pietro Giannone schreiben über ihn in ihren Autobiographien. Ausführlich berichtet über sein Leben, seine Schriften und hinterlassenen Manuskripte R. Ajello im «Dizionario biografico degli Italiani» (Rom $1960 \mathrm{ff}$.).

${ }^{3}$ Natale Lettieri veröffentlichte eine «Dissertatio de remedio febrifugo, nostrate cortici Peruviano pari, vel forsan eo praestantiori; cui accedit appendix de balneorum usu in febribus essentialibus» (Neapel 1784, $118 \mathrm{~S}$.).

4 Nicola Braucci (1719-1774) studierte in Neapel Medizin und durchreiste ganz Italien, Pflanzen und Mineralien sammelnd. Er war Arzt und seit 1754 Professor für Naturgeschichte in Neapel. Mit Cirillo bewarb er sich 1760 um den Lehrstuhl für Botanik, unterlag jedoch, da er noch Tournefort anhing, während Cirillo bereits das System Linnés vertrat. 
Ein späteres Werk, das Manuskript blieb, «Istoria naturale della Campania sotterranea», erweist ihn als bedeutenden Geologen Kampaniens. Nähere Auskunft gibt U. Baldini im «Dizionario biografico degli Italiani» (Rom $1960 \mathrm{ff}$.).

5 Vincenzo Petagna (1734-1810), aus Neapel, Verfasser der Werke «Institutiones botanicae» (Bd.1: De philosophia botanica, Neapel 1785; Bde.2-5: De plantis in specie, Neapel 1787), «Specimen insectorum ulterioris Calabriae» (Frankfurt 1787; neue Ausgabe Leipzig 1820) und «Institutiones entomologicae» (2 Bde., Neapel 1792).

${ }^{6}$ Joseph Pitton de Tournefort (1656-1708), Professor am Jardin du Roi in Paris, großer Systematiker. Textband und 2 Tafelbände: «Elémens de botanique, ou méthode pour connaître les plantes» (Paris 1694); erweiterte 2. Auflage in lateinischer Sprache: «Institutiones rei herbariae» (Paris 1700); 3. Auflage von Antoine de Jussieu (Lyon 1719).

${ }^{7}$ Francesco Serao (1702-1783) bekleidete als trefflicher Erzieher der Reihe nach die Lehrstühle für Anatomie, theoretische und praktische Medizin in Neapel. Er war Anhänger Boerhaaves und Iatromechanist. Sein Bericht über einen heftigen Vesuvausbruch, «Istoria dell'incendio del Vesuvio accaduto nel mese di maggio dell'anno 1737» (Neapel 1738) wurde auch ins Lateinische und Französische übersetzt. In «Lezioni academiche sulla tarantola» bestritt er 1742 das Vorurteil, daß allerlei Krankheiten auf dem Stich der Tarantel beruhen. 1757 übersetzte er Pringles Buch über die Soldatenkrankheiten. Noch 1780 veröffentlichte er einen Index erprobter Medikamente.

${ }^{8}$ Sir John Pringle (1707-1782), einer der großen Ärzte seiner Zeit. Statt als Kaufmann kehrte er als Schüler Boerhaaves von Holland heim. Er wurde Professor in Edinburg, dann aber Armeearzt, 1749 Praktiker in London, 1763 Leibarzt, 1766 geadelt. 1772-1778 war er Präsident der Royal Society. Seine «Observations on the diseases of the army» (London 1752) wurden in verschiedenen Sprachen oft aufgelegt.

${ }^{9}$ Bruno Amantea (1750-1819), Arztsohn aus Grimaldi bei Cosenza, wurde im Jesuitenkollegium zu Cosenza erzogen. In Neapel studierte er Medizin. Sein Fleiß fiel auf. Das Beste gaben ihm Cotugno in Anatomie und Cirillo in Geburtshilfe. Er promovierte 1773 und wurde 1776 Chirurg, später Chefchirurg am großen Spital degli Incurabili. Als Anatomieprofessor lehrte er die Anwendungen der Anatomie auf die Chirurgie. Er gewann Ruf als Chirurg und Geburtshelfer. Über ihn: Bruno Magliari, Elogio del cavaliere Bruno Amantea, Aversa 1820.

10 Domenico Cotugno (1736-1822) von Ruvo di Puglia wirkte wie Cirillo an der Universität und am Ospedale degli Incurabili in Neapel. Als entdeckender Anatom, denkender Physiologe, beobachtender Pathologe war er vielseitig, scharfsinnig, bahnbrechend. Luigi Belloni hat in Spezialarbeiten den Neurologen und Gewebepathologen gewürdigt. Siehe Artikel und Literaturhinweise im «Dictionary of Scientific Biography» (New York $1970 \mathrm{ff}$.).

${ }^{11}$ Francesco Conforti (1743-1799), aus Calvanico (Salerno), erweckte durch ein theologisches Buch die Aufmerksamkeit des Ministers Bernardo Tanucci und wurde Universitätsprofessor für Geschichte und Hoftheologe in Neapel. Auch war ihm das Amt aufgetragen, ausländische Bücher zu zensieren. Da er es zu großzügig ausübte, wurde er 1796 seiner Stellen enthoben und eingekerkert. Zur Zeit der Republik wurde er Innenminister, nach deren Zusammenbruch wieder verhaftet und am 7. Dezember 1799 hingerichtet. - Literaturhinweise in der «Enciclopedia Italiana».

12 Angelika Kauffmann (1741-1807), vor allem Bildnismalerin. Sie lebte mit ihrem Gatten, dem Maler Antonio Zucchi, seit 1782 in Rom. Ihr Geburtshaus in Chur trägt eine Gedenktafel. 
${ }^{13}$ Francesco Filomena veröffentlichte die Abhandlungen: «Breve saggio sull'operazione dell'oppio, e dell'aria fissa, ed infiammabile negli animali, secondo il sistema dell'elettricità» (Neapel 1781, 88 S.); «Saggio critico e filosofico su i veleni, con un applicazione alla lue venerea ed al vajuolo» (Neapel 1804, $42 \mathrm{~S}$.); «Diverse istruzioni della natura e prospetto fisiologico de' muscoli, dei nervi del sesto pajo e del meccanismo della respirazione» (Neapel 1805,63 S.).

${ }^{14}$ Matteo Tondi (1762-1835), Generalinspektor der Gewässer und Wälder im Königreich Neapel, dann Professor der Oryktognosie (Mineralogie) in Neapel. Schon mit 24 Jahren schrieb er «Istituzioni di chimica» (Neapel 1786). Zu den späteren Werken gehören die umfangreichen «Elementi di orittognosia» (3 Bde., Neapel 1817) und «La scienza selvana ad uso de' forestali» (3 Bde., Neapel 1821).

15 Der Botaniker Gaetano Nicodemi starb 1804 in Lyon.

${ }^{16}$ Peder Ascanius (1723-1803), norwegischer Zoologe und Mineraloge, Schüler Linnés 1753, lehrte Naturgeschichte in Kopenhagen und war Assessor am Oberbergamt. Er bereiste 1768-1770 die Küste Norwegens. Sein Hauptwerk sind die «Icones rerum naturalium, ou figures enluminées d'histoire naturelle du nord» (5 Teile in 4 Bänden, Kopenhagen 1772-1805).

${ }_{17}$ Johann Anders Murray (1740-1791) aus Stockholm, Professor der Medizin und Direktor des botanischen Gartens in Göttingen, Übersetzer wichtiger Werke schwedischer Ärzte, bedeutender Botaniker und Pharmakologe. Linné hat nach ihm die ostindische Pflanzengattung «Murraya» benannt, sein Schüler dafür zwei Ausgaben des «Systema vegetabilium» Linnés betreut und viele neue Pflanzen beschrieben. Murrays umfassendes Werk über pflanzliche Heilmittel hieß «Apparatus medicaminum» (6 Bde., Göttingen 1776-1792); es erschien auch deutsch als «Arzneyvorrath».

${ }^{18}$ Ferrante Imperato (1550-1625), Apotheker in Neapel. Sein Name steht auf dem Foliowerk «Dell' historia naturale libri XVIII» (Neapel 1599, 791 S.; 2. Auflage Venedig 1672). Lateinische Ausgabe: Köln 1695.

${ }^{19}$ Francesco Caracciolo (1752-1799), der größte neapolitanische Admiral des 18. Jahrhunderts. Er wurde 1779 von Acton ausgeschickt, um sich auf englischen Schiffen im Krieg gegen Amerika einzuüben. Nach seiner Rückkehr 1781 kämpfte er jahrelang gegen algerische und tunesische Freibeuter. Sein Ruf als Befehlshaber wuchs. Als die königliche Familie am 23. Dezember 1798 nach Palermo flüchtete, begleitete er sie, mußte aber erkennen, daß die Engländer den Hof beherrschten. Er kehrte nach Neapel zurück und übernahm das Kommando über die kleine Flotte der Republik. Nach dem Umschwung wollte Ruffo ihn retten, hatte aber nichts mehr zu sagen. Näheres in der «Enciclopedia Italiana», Bd. 8, Mailand 1930, S.928.

${ }^{20}$ Ferdinand IV. (1751-1825) kam schon 1759 auf den Thron Neapels. Lange leitete der erfahrene Minister Bernardo Tanucci die Regierung. Die herrschsüchtige Königin Karolina, eine Tochter Maria Theresias, erreichte 1777 die Entlassung des Greises. Dafür ließ sie den als Admiral berufenen Engländer Acton aufsteigen. Englands Gesandter seit 1764, Sir William Hamilton (1730-1803), als Vulkanologe, Altertumsforscher in Herkulaneum und Pompeji, Sammler antiker Vasen und Fachschriftsteller ehrenwert, geriet seinerseits unter den Einfluß einer Frau, die er 1791 heiratete. Lady Emma, die Vertraute Karolinas, erwirkte durch ihren Mann 1793 den Bund Neapels mit England. Als das von Steuern gedrückte Volk sich erhob, flüchtete Ferdinand Ende 1798 nach Palermo. Fabrizio Ruffo 
(1744-1827) von San Lucido (Provinz Cosenza), seit 1791 Kardinal, sammelte im Auftrag Ferdinands ein Heer und stürzte im Juni 1799 die Republik. Nelson half zur See mit und wurde Lady Hamiltons Geliebter. Er und Acton warben um höhere Gunst als Vollstrecker der Rache. - England und Österreich halfen Ferdinand auch über die Absetzung von 1805-1814 und über die Revolution von 1820 hinweg, so daß er bei seinem Tode absoluter König beider Sizilien war. - Das Werk des Historikers Vincenzo Cuoco oder Coco (1770-1823) heißt «Saggio storico sulla rivoluzione napoletana del 1799» und erschien 1801.

${ }^{21}$ Arturo Castiglioni (1874-1953), einer der größten Medizinhistoriker Italiens, wäre beinahe vom gleichen Schicksal ereilt worden wie Cirillo. Der glühende Patriot mußte 1938 sein Vaterland verlassen. Seine Bibliothek und Kunstsammlung wurden geplündert, seine Werke eingestampft. Er überlebte den Krieg in Amerika und kehrte 1946 zurück. Seine «Storia della Medicina», bis heute das italienische Hauptwerk für allgemeine Medizingeschichte (1.Auflage Mailand 1927, 4. Auflage in zwei Bänden 1948), enthält über Cirillo schon 1927 die Worte: «Fra i medici italiani più illuminati e più colti va nominato Domenico Cirillo, napoletano (1739-1799), una delle più belle e gloriose figure della medicina italiana.» In der Auflage von 1936 fügt er hinzu: «Egli fu un uomo di grandissimo ingegno, oratore eloquente e ferventissimo patriota che prese parte attiva alle cospirazioni contro il malgoverno napoletano.» Dann würdigt er seinen Kampf für die Gefängnis- und Spitalreform. Als hervorragender Botaniker und gelehrter Arzt sei er «veramente un precursore della nuova medicina». Heldenhaft habe er das Gerüst zur Hinrichtung bestiegen.

\section{Bibliographie}

\section{Botanische Schriften Domenico Cirillos}

- Ad botanicas institutiones introductio, Neapel 1766 (28 S. und 2 Tafeln); 2. Auflage 1771.

- A letter to William Watson, giving some account of the Manna tree and of the tarantula. In: Philosophical Transactions 60 (1770), S.233-238.

- De essentialibus nonnullarum plantarum characteribus commentarius, Neapel 1784 (75 S. und 4. Tafeln).

- Fundamenta botanica sive philosophiae botanicae explicatio, 3. vermehrte Auflage, 2 Bände, Neapel 1785-1787 (516 + 506 S., Index und 2 Tafeln).

- Plantarum rariorum regni Neapolitani fasciculus primus et secundus, Neapel 1788-1792 (folio, $39+35$ S. und je 12 farbige Tafeln). - Mit vielen Gartenpflanzen. Neuausgabe des Textes des zweiten Faszikels in Paulus Usteri, Annalen der Botanik, 13. Stück, S.44-65. Beschreibung und Abbildung einiger seltener oder ganz neuer Arten. Zum dritten Faszikel lagen der Text und eine Tafel bereit.

- Tabulae botanicae elementares quatuor priores sive Icones partium quae in fundamentis botanicis describuntur, Neapel 1790 (folio minor, 18 S. und 4. Tafeln). - Mit klarem Hinweis auf die befruchtende Wirkung des Blütenstaubs.

- Cyperus Papyrus, Parma 1796 (folio maxima, 20 S. und 2 farbige Tafeln). 


\section{Medizinische Schriften Domenico Cirillos}

- Nosologiae methodicae rudimenta, Neapel 1780.

- Aviso intorno alla maniera di adoperare l'unguento di sublimato corrosivo nella cura delle malattie veneree, Neapel 1780.

- Deutsche Ausgabe: Nachricht von dem Gebrauch einer Salbe mit dem Quecksilbersublimat in venerischen und andern solchen Krankheiten, die oft den besten Mitteln widerstehen. In: Sammlung auserlesener Abhandlungen zum Gebrauche praktischer Aerzte, Bd.8, Leipzig 1783, S. 526-551.

- Französische Ausgabe: Avis au public sur la manière d'employer l'onguent de sublimé corrosif dans le traitement des maladies vénériennes, et d'autres maladies qui résistent souvent aux meilleurs remèdes. Traduit par Claude-François Duchanoy. In: Journal de médecine, chirurgie, pharmacie... 59 (Paris 1783), S. 506-526.

- Osservazioni pratiche intorno alla lue venerea, Neapel 1783, XVIII + 288 S. Dasselbe: Venedig 1786, XVI + 288 S. Neuausgabe 1800. - Die Bemerkungen über Eingeweidesyphilis stützt Cirillo auf Leichenbefunde.

- Deutsche Ausgabe: Praktische Bemerkungen über die venerischen Krankheiten, nebst einer ausführlichen Anweisung, dieselbe durch Sublimateinreibungen gründlich zu heilen. Aus dem Italiänischen übersetzt und mit Zusätzen und Anmerkungen vermehrt von Johann Gottlieb Dähne. Leipzig 1790 (XIV + 450 S.). Dasselbe: Wien 1791 (XVI + 495 S.).

- Französische Ausgabe: Traité complet et observations pratiques sur les maladies vénériennes, ou nouvelle méthode de guérir radicalement la syphilis la plus invétérée. Traduit de l'italien, avec des notes, par Charles-Edouard Auber. Paris 1803 (XX + 395 S.).

- Discorsi accademici, Neapel 1789 (335 S.).

- Metodo di amministrare la polvere antifebbrile del dottor James, Neapel 1794 (120 S.).

(Robert James, 1703-1776, ein englischer Arzt, Verfasser eines «Medical dictionary», einer verbreiteten Schrift über Tollwut und anderer praktischer Anweisungen, war der Erfinder des erfolgreichen James' Powder, eines fiebersenkenden Antimonpräparates.)

- Dominicus Cyrillus, Tractatus de pulsibus, Neapel 1802 (139 S.).

- Domenico Cotugno e Domenico Cirillo, Alcuni scritti inediti, Pisa 1890 (28 S.).

Die klinischen Tagebücher (2 Manuskriptbände) sind in der Biblioteca di S. Martino, die mit der Biblioteca Vittorio Emanuele III verbunden wurde, in Neapel aufbewahrt.

\section{Schriften zur Hygiene, Entomologie und Pharmakologie}

Cirillo veröffentlichte hygienische Untersuchungen über die Gerbereien, deren Unschädlichkeit er nachwies (Neapel 1785), ebenso Pläne zur Reform der Spitäler und Gefängnisse (Nizza 1787).

- Entomologiae neapolitanae specimen primum, Neapel 1787 (15 S. und 12 farbige Tafeln).

- Materia medica regni mineralis, Neapel 1792 (XXIII + 132 S.). - Untersuchungen über Arzneimittelwirkung.

- Materia medica regni animalis. Erst 1861 von Giuseppe Maria Carusi, dem Sohn eines Schülers von Cirillo, veröffentlicht. - Die «Materia medica vegetabilis» findet sich in Band 2 der «Fundamenta botanica». 


\section{Literatur über Domenico Cirillo}

- Atto Vannucci, I Martiri della libertà italiana dal 1794 al 1848, 3. Auflage, Florenz 1860.

- M.D'Ayala, Vita di Domenico Cirillo. In: Archivio storico italiano, Serie terza, XI (1870), parte seconda, S. 106; XII (1870), parte prima, S. $106 \mathrm{ff}$.

- J.Petersen, Domenico Cirillo og den Partenopaeiske Republik. In: Ugeskrift for Laeger, 5.R., VII (Kopenhagen 1900), S.673; 697.

- Comitato Napoletano per le onoranze centenarie a Domenico Cirillo, Neapel 1901.

- Andrea Ferrannini, Un naturaliste-medico martire di libertà. In: Bollettino della Società Eustachiana 15, no. 4 (Camerino 1917), S.1-15.

- Andrea Vitelli, Domenico Cirillo nella storia delle riforme sociali del secolo XVIII. In: Gazzetta internazionale di medicina 21 (Napoli 1918), S.11-16.

- Giovanni Battista de Toni, Appunti dal carteggio inedito di Domenico Cirillo. In: Rivista di storia delle scienze mediche e naturali 16 (Florenz 1925), S. 193-195.

- Benedetto Croce, La rivoluzione napoletana del 1799, Bari 1926, S. 249 ff.

- Artikel über Cirillo in «La grande Encyclopédie» und in der «Enciclopedia Italiana», Bd.10, Mailand 1931.

\section{Summary}

The author, former director of the Swiss School in Naples and lecturer for German literature at the University of that town, has always been interested in great figures of the natural sciences. His essay about Domenico Cirillo does not analyse his scientific work, but tries to give a true picture of his life and of the development of this fascinating personality.

Prof. Dr. Johann Ulrich Marbach

Ostring 17

CH-3006 Bern 